To evaluate the effect of disease activity on accrual damage.

Methods: A retrospective study was carried out from January 2010 to December 2018. Clinical records of patients with SLE (ACR criteria 1982/97) were reviewed considering baseline visit as the first clinical or control visit of 2010 . For subsequent visits, data were collected annually until 2018. SLE activity was defined for each visit according to GLADEL's definition: 1- Remission Without Treatment (RwT): SLEDAI 0, without prednisone or immunosuppressive drugs (IS); 2- Remission on Treatment (RoT): SLEDAI 0, prednisone up to $5 \mathrm{mg} /$ day or immunosuppressive drugs in maintenance doses; 3- LDAS (Low Disease Activity Status): SLEDAI $\leq 4$, prednisone up to $7.5 \mathrm{mg} /$ ay and/or IS in maintenance doses; 4- Non-Optimal Activity Control (NOC): SLEDAl> 4, prednisone> $7.5 \mathrm{mg} /$ day and/or IS in induction dose. The use of hydroxychloroquine was allowed for all groups. For the analysis, patients who remained in remission (with and without treatment) or LDAS for at least $75 \%$ of the follow-up time were grouped and compared with patients who remained active during that same period. Demographic, laboratory, treatment related variables and death were studied. Accrual damage was assessed with SLICC / SDI. Patients with less than two annual visits were excluded.

Statistical analysis: descriptive measures, Test T, Mann Whitney, Chi2 Test, Fisher's exact test, bivariate correlation, logistic regression model with mixed effects.

Results: Two hundred eighty-five medical records were reviewed and 100 patients with SLE were included, $89 \%$ women, mean age at baseline visit $38.5 \pm 12$ years old and mean time of disease $9.3 \pm 7.3$ years. The average SLEDAI and SLICC/ SDI baseline scores were 3.7 and 0.8 respectively. The SLICC/ SDI score at last visit was 2.2 and the average SLICC/SDI change ( $\triangle$ SLICC) compared to baseline visit score was $1.4 \pm 1.6$.

The prevalence of patients who were in remission for at least $75 \%$ of the follow-up time was $38 \%$ [95\% Cl 26.6, 45.4]. NOC patients categorized at baseline visit had the highest $\triangle$ SLICC (p 0.0001). The $\triangle$ SLICC was significantly lower in patients who were at least $75 \%$ of the follow-up time in remission ( $p$ 0.01) or LDAS ( $p$ 0.01) compared to those with NOC. In the Logistic Regression Model, the chance of changing the SLICC/SDI score was 2.9 times higher for the NOC group than for RwT.

Conclusion: The frequency of remission in this cohort of patients with SLE was $38 \%$.

Worse control of disease activity, was associated with higher accumulated damage.

Disclosure of Interests: None declared

DOI: 10.1136/annrheumdis-2020-eular.6062

\section{FRI0607-HPR FREQUENCY AND PATIENTS BELIEFS ON VACCINATION IN RHEUMATIC DISEASES}

M. Viola ${ }^{1}$, A. Benitez ${ }^{1}$, C. Garbarino ${ }^{1}$, G. Rodriguez ${ }^{1}$, F. Benavidez ${ }^{1}$, C. Peon ${ }^{1}$, E. S. Blanco ${ }^{1}$, H. Molina ${ }^{1}$, G. Gómez ${ }^{1}$, G. Redondo ${ }^{1}$, M. Delavega ${ }^{1}$, D. Mata ${ }^{1}$, A. Riopedre ${ }^{1}$, O. Messina ${ }^{1} .{ }^{1}$ Hospital General de Agudos Dr. Cosme Argerich, Ciudad Autónoma de Buenos Aires, Argentina

Background: Infectious diseases are increased in patients with rheumatic disorders; vaccination improves morbidity and mortality

Objectives: The aim of this study was to describe the frequency of vaccination in patients with rheumatic disorders and to compare the results with those obtained in 2009 and 2013 in a similar population. We also identified factors leading to lack of vaccination and patients beliefs on vaccines.

Methods: Multicentric cross sectional study in patients with autoinmune diseases from external rheumatology offices. Evaluation of vaccination status and patients knowledge about vaccines were studied. A comparative analysis was carried out with the series registered in 2009 and 2013 in a similar population.

Results: 179 patients (158 female, $88.3 \%$ and 21 male, $11.7 \%$ ) were evaluated. Median age was 52 years. Main pathologies were: Rheumatoid Arthritis $65.9 \%$ (n:118), Systemic Lupus Erythematosus 11.7\% (n:21), Systemic Sclerosis 3.9\% (7), Sjogren Syndrome $n=3.4 \%$ (n:6), other diseases 15\% (n: 27). Median disease duration: 8.87 years. Ninety three percent of patients $(n: 167)$ were taking inmunomodulators and $36.8 \%$ (n: 66) were using oral corticosteroids (20mg/ day or less); $26,8 \%$ patients ( $\mathrm{n}: 48$ ) were receiving biological therapies. Vaccination frequency in the population was: Influenza $82 \%$ (147); 13-valent conjugate pneumococcal $69.3 \%$ (124), 23-valent pneumococcal $64.2 \%$ (115) and hepatitis B 62\% (111). Comparative with 2009 and 2013 series there was an increase in the rate of vaccinated patients: influenza ( $82 \%$ vs. $39,1 \%$ and $74,2 \%$ respectively), antineumococcal (64\% vs. $17 \%$ and $29 \%)$ and hepatitis B (62\% vs. $6,7 \%$ and $26,7 \%$ )

Reasons for non-vaccination were absence of medical indication ( $41 \%$ of patients for hepatitis B; $32 \%$ for 23 -valent pneumococcal; $38 \%$ for 13 -valent pneumococcal and $34 \%$ for influenza).
139 patients $(77,7 \%)$ knew the benefits of vaccines, $164(91,6 \%)$ thought vaccines are useful; $134(74,9 \%)$ reported that vaccines may decrease dying probability, $155(86,5 \%)$ thought that vaccines are effective to prevent diseases and 149 patients $(83,2 \%)$ believed that they prevent serious infections. 71 patients (39\%) believed that vaccines can lead to serious consequences and $99(55,3 \%)$ that they are more likely to acquire infections than the rest of the population.

Conclusion: Frequency of vaccination has increased since 2009 but there is still misinformation regarding vaccines risks and benefits. Promotion and information is essential to improve adherence.

\section{References:}

[1] 2019 update of EULAR recommendations for vaccination in adult patients with autoimmune inflammatory rheumatic diseases. Furer V, et al. Ann Rheum Dis 2020;79:39-52

[2] Vaccines and Disease-Modifying Antirheumatic Drugs: Practical Implications for the Rheumatologist. Friedman MA et al. Rheum Dis Clin North Am. 2017 Feb; 43 (1):1-13.

[3] Recommendations and barriers to vaccination in systemic lupus erythematosus. Garg M et al. Autoimmun Rev. 2018 Oct; 17 (10):990-1001.

[4] Comparison of national clinical practice guidelines and recommendations on vaccination of adult patients with autoimmune rheumatic diseases. Papadopoulou D. et al. Rheumatol Int. 2014 Feb;34 (2):151-63.

[5] Guías de recomendaciones de prevención de infecciones en pacientes que reciben modificadores de la respuesta biológica. Jordán R. Et al. Rev Arg Reumatol. 2014; 25 (2): 08-26.

Disclosure of Interests: Malena Viola: None declared, Alejandro Benitez: None declared, Cecilia Garbarino: None declared, Gonzalo Rodriguez: None declared, Federico Benavidez: None declared, Claudia Peon: None declared, Eliana Soledad Blanco: None declared, Hernan Molina: None declared, Gimena Gómez: None declared, griselda redondo: None declared, Maria DeLaVega: None declared, Dario Mata: None declared, Augusto Riopedre: None declared, Osvaldo Messina Speakers bureau: Amgen; Americas Health Foundation; Pfizer DOI: 10.1136/annrheumdis-2020-eular.4986

\section{FRI0608-HPR SEVERITY AND PREDICTORS OF PAIN INTENSITY AND HAND DISABILITY IN PATIENTS WITH TRAPEZIOMETACARPAL OSTEOARTHRITIS}

T. Hamasaki $^{1,2,3}$, P. Harris ${ }^{1,2}$, N. Bureau ${ }^{1,2,3}$, N. Gaudreault ${ }^{4,5}$ N. Patenaude ${ }^{4,5,6}$, M. Choinière ${ }^{2,3} .{ }^{1}$ CHUM Hospital (Université de Montréal), Montréal, Canada; ${ }^{2}$ Université de Montréal, Montreal, Canada; ${ }^{3}$ Centre de recherche du CHUM (CRCHUM), Montreal, Canada; ${ }^{4}$ Université de Sherbrooke, Sherbrooke, Canada; ${ }^{5}$ Centre de recherche du CHUS (CRCHUS), Sherbrooke, Canada; ${ }^{6} \mathrm{CHUS}$ Hospital (Université de Sherbrooke), Sherbrooke, Canada

Background: Trapeziometacarpal osteoarthritis (TMO) is one of the most prevalent and painful forms of hand osteoarthritis. ${ }^{1}$ It limits thumb mobility, ${ }^{2}$ reduces hand functions, and manual activities. ${ }^{1}$ Yet, no study has exhaustively documented the characteristics of this pathology using a biopsychosocial approach (e.g., pain, disability, psychological well-being, pain-related catastrophic thinking, quality of life). Furthermore, radiographic TMO severity and symptomatology are only weakly to moderately correlated. ${ }^{3,4}$ The extent to which biopsychosocial factors (e.g., pain duration, depression, education) contribute to interindividual variability in TMO pain and hand disability merits further investigation.

Objectives: This study aimed at 1) describing the pain experience of patients with trapeziometacarpal osteoarthritis (TMO) from a biopsychosocial perspective, and 2) identifying predictors of their pain intensity and hand disability.

Methods: A total of 227 TMO patients recruited from 16 healthcare institutions completed validated questionnaires assessing their biopsychosocial characteristics. The associations of pain severity and hand disability with various biopsychosocial characteristics were analyzed by linear regression.

Results: The participants' mean age was $62.6 \pm 8.5$ years and $78 \%$ were women Their mean pain intensity on the average in the last seven days was $5.8 \pm 2.1$ while their hand disability scores averaged $45.4 \pm 18.8$ on the QuickDASH. In terms of health-related quality of life, the participants' scores on the physical and mental summary scales of the SF-12v2 were $41.0 \pm 9.4$ and $48.7 \pm 9.7$ respectively. Results of the multivariable linear regression analyses revealed that age living condition, pain frequency, pain-related catastrophic thinking, and depression levels accounted for $43.3 \%$ of the variance in pain intensity while age, sex, pain intensity, pain-related catastrophic thinking, depression, level of education, employment status and living condition accounted for $60.6 \%$ of the variance in hand function.

Conclusion: This comprehensive study showed that patients with TMO experience pain of moderate to severe intensity which can affect various aspects 